International Mathematical Forum, Vol. 9, 2014, no. 26, 1253 - 1259

HIKARI Ltd, www.m-hikari.com

http://dx.doi.org/10.12988/imf.2014.46112

\title{
Extensions of Laplace Type Problems \\ in the Euclidean Space
}

\author{
Giuseppe Caristi \\ Univerisity of Messina, Department S.E.A.M. \\ Via dei Verdi, 75, 98122 - Messina, Italy \\ Marius Stoka \\ Accademia delle Scienze di Torino, Italy
}

Copyright (c) 2014 Giuseppe Caristi and Marius Stoka. This is an open access article distributed under the Creative Commons Attribution License, which permits unrestricted use, distribution, and reproduction in any medium, provided the original work is properly cited.

\begin{abstract}
The classical Buffon needle problem is to find the probability that a needle of length $n$ when dropped on a floor made of boards of width $b$ will cross a crack between the boards. This problem can be solved by evaluating a simple single integral. In his extension of the problem, Laplace considered a floor tiled by congruent rectangles and considered the probability of the needle crossing one or two of the cracks bettween the rectangles. In 1974 M. Stoka studies an extension of the BuffonLaplace needle problem in the space $R^{n}$. In this paper we consider two extension of the Laplace problem in $E_{3}$
\end{abstract}

Keywords: Geometric Probability, stochastic geometry, random sets, random convex sets and integral geometry

\section{The Buffon-Laplace needle problem}

In Buffon's original work the particle was considered to have length $l$ and the mesh separation distance $d[2,3]$. Henceforth all lengths are in units of the line spacing $d$. The solution is found by considering only two lines, one at 
$y=0$ and the other at $y=1$, and the needle's center of mass may lie at any height between 0 and 1 . The needle orientation takes any angle $\theta \in \pm \pi / 2$ with uniform probability. An intersection is avoided when the center of mass position is within $y \in[l|\sin \theta| / 2,1-l|\sin \theta| / 2]$. Averaging this range over all orientations results in the intersection probability

$$
P_{\text {int }}(l>1)=1-\frac{1}{\pi} \int_{-\pi / 2}^{\pi / 2}(1-l|\sin \theta|) d \theta=\frac{2 l}{\pi} .
$$

When the needle length equals the line spacing $(l=1)$ the intersection probability is $2 / \pi=0.637$, indicating non-intersecting orientations about $\theta=0$. The solution for $l>1$ is found by restricting the range in $\theta$ to $\theta \in \pm \arcsin (1 / l)$, with intersection probability

$$
\begin{aligned}
P_{\text {int }}(l>1)= & -\frac{1}{\pi} \int_{-\arcsin (1 / l)}^{\arcsin (1 / l)}(1-l|\sin \theta|) d \theta= \\
& \frac{2}{\pi}\left(l-\sqrt{l^{2}-1}+\sec ^{-1} l\right) .
\end{aligned}
$$

This expression has the correct large $l$ asymptote of $P(l \rightarrow \infty)=1$. This analysis has been used to find experimental values for $\pi$ by dropping needles and counting the intersections, with at least one suspected case of fraud [1].

While Laplace's generalization of Buffon's work [5, 6] was rectangular meshes of arbitrary length and width, we only consider the square mesh of sidelength. The center of mass samples an allwable $A(\theta)=(1-l \cos \theta)(1-l|\sin \theta|)$, with the result that

$$
P_{\text {int }}(l>1)=1-\frac{1}{\pi} \int_{-\pi / 2}^{\pi / 2} A(\theta) d \theta=\frac{l(4-l)}{\pi} .
$$

It does not appear that Laplace calculated the probabilities for longer lengths, although it is clear that not until $l=\sqrt{ } 2$ is an intersection guaranteed. Changing the limits in the integral in equation (4) to $\pm \arcsin (1 / l)$ (as in equation (2)) results in an intersection probability of

$$
P_{\text {int }}(l>1)=1-\frac{1}{\pi} \int_{-\arcsin (1 / l)}^{\arcsin (1 / l)} A(\theta) d \theta=
$$




$$
1-\frac{1}{\pi}\left[\arcsin (1 / l)-\arccos (1 / l)+2 \sqrt{l^{2}-1}-\frac{l^{2}}{2}-1\right]
$$

This expression has the correct limit that $P_{\text {int }}(l=\sqrt{2})=1$.

To make the Buffon-Laplace results applicable to actual particles, we first generalize the analysisto spherocylinders [7] - cylinders (dimensionless diameter $\sigma)$ with hemispherical endcaps. All points on the surface of the spherocylinder are a distance $\sigma / 2$ from the central line segment. The total length of the spherocylinder is $l+\sigma$ and its aspect ratio $1+l / \sigma$, with $l=0$ corresponding to a sphere. The finite particle width reduces the total area available to the center of mass to

$$
A(\theta, \sigma)=(1-\sigma-l \cos \theta)(1-\sigma-l|\sin \theta|)
$$

and the intersection probability is found to be

$$
P_{\text {int }}(l<1)=\frac{4 l-l^{2}}{\pi}+\frac{\sigma}{\pi}[(2 \pi-4)-\sigma(\pi-3)-2 l] .
$$

Now we considering the Stoka paper [8] we prove two anothers extensions.

\section{First extension}

We consider a lattice $\Re\left(a_{1}, a_{2}, a_{3} ; \lambda\right)$ with foundamental cell $\mathcal{P}_{0}$ a parallelepiped with sides $a_{1}, a_{2}, a_{3}$ e angolo $\left.\left.\lambda \in\right] 0, \frac{\pi}{2}\right]$.

We have that:

$$
\operatorname{vol} \mathcal{P}_{0}=a_{1} a_{2} a_{3} \sin \lambda .
$$

Let $s$ a random segment of constant lenght $l$ with $l<\inf \left(a_{1}, a_{2}, a_{3}\right)$.

Our aim is to compute the probability that this segment intersects a side of lattice $\Re$.To be more specific, we denote by $O$ the center of $s$, the position of the segment is determineted by the center and the direction cosines of the line support:

$$
\alpha_{1}=\cos \theta, \alpha_{2}=\sin \theta \cos \varphi, \alpha_{3}=\sin \theta \sin \varphi .
$$

To compute the probability $P_{\text {int }}$ we consider the parallelepiped $\mathcal{P}_{0}^{*}$ with a vertix in $A\left(\frac{l}{2} \cos \theta, \frac{l}{2} \sin \theta \cos \varphi, \frac{l}{2} \sin \theta \sin \varphi\right)$, with the sides parallel to the sides of $\mathcal{P}_{0}$ and with sides

$$
a_{1}-l \cos \theta, a_{2}-l \sin \theta \cos \varphi, a_{3}-l \sin \theta \sin \varphi,
$$

then 


$$
\operatorname{volP}_{0}^{*}=\left(a_{1}-l \cos \theta\right)\left(a_{2}-l \sin \theta \cos \varphi\right)\left(a_{3}-l \sin \theta \sin \varphi\right) \sin \lambda .
$$

Denoting by $M_{1}$ the set of all segments $s$ which have their center in $\mathcal{P}_{0}$. Denote likewise by $N_{1}$ the set of all segments $s$ in completely contained in $P_{0}$. In view of [8]:

$$
P_{\text {int }}=1-\frac{\mu\left(\mathcal{N}_{1}\right)}{\mu\left(\mathcal{M}_{1}\right)},
$$

where $\mu$ is the Lebesgue measure in the space $E_{3}$.

We compute the measures $\mu\left(\mathcal{M}_{1}\right)$ and $\mu\left(\mathcal{N}_{1}\right)$ using the cinematic measure of Blaschke in the space $E_{3}[2]$.

$$
d k=d \psi \wedge d \varphi \wedge \sin \theta \wedge d \theta \wedge d x \wedge d y \wedge d z
$$

where $x, y, z$ are the coordinates of the center of $s, \varphi$ and $\theta$ are the angles and $\psi$ the rotation angle.

We have

$$
0 \leq \varphi, \theta \leq \frac{\pi}{2}, 0 \leq \psi \leq 2 \pi
$$

By (8), (11) and (12) we have that

$$
\mu\left(\mathcal{M}_{1}\right)=\int_{0}^{2 \pi} d \psi \int_{0}^{\frac{\pi}{2}} d \varphi \int_{0}^{\frac{\pi}{2}} \sin \theta d \theta \iiint_{\left\{(x, y, z) \in \mathcal{P}_{0}\right\}} d x d y d z=\pi^{2} a_{1} a_{2} a_{3} \sin \lambda
$$

Considering (9), we have

$$
\begin{gathered}
\mu\left(\mathcal{N}_{1}\right)=\int_{0}^{2 \pi} d \psi \int_{0}^{\frac{\pi}{2}} d \varphi \int_{0}^{\frac{\pi}{2}} \sin \theta d \theta \iiint_{\left\{(x, y, z) \in \mathcal{P}_{0}^{*}\right\}} d x d y d z= \\
2 \pi \sin \lambda \int_{0}^{\frac{\pi}{2}} \int_{0}^{\frac{\pi}{2}} \sin \theta\left(a_{1}-l \cos \theta\right)\left(a_{2}-l \sin \theta \cos \varphi\right)\left(a_{3}-l \sin \theta \sin \varphi\right) d \theta d \varphi= \\
2 \pi \sin \lambda\left[\frac{\pi}{2} a_{1} a_{2} a_{3}-\frac{\pi l}{4}\left(a_{1} a_{2}+a_{1} a_{3}+a_{2} a_{3}\right)+\frac{l^{2}}{3}\left(a_{1}+a_{2}+a_{3}\right)-\frac{l^{3}}{8}\right] .
\end{gathered}
$$


By cobining (10), with (13) and (14) we obtain that

$$
P_{\text {int }}=\frac{l}{\pi a_{1} a_{2} a_{3}}\left[\frac{\pi}{2}\left(a_{1} a_{2}+a_{1} a_{3}+a_{2} a_{3}\right)-\frac{l}{3}\left(a_{1}+a_{2}+a_{3}\right)-\frac{l^{2}}{8}\right] .
$$

This probability is indipendent by $\lambda$, i.e. is equal with the probability for a right parallelepiped, that was find in a previous paper [8].

Now we want to show that if the sides $a_{1}, a_{2}, a_{3}$ and $l$ multiply with the same factor $\mu$, the probability $P_{\text {int }}$ is unchanged.

In fact, if replacing

$$
a_{1}, a_{2}, a_{3}, l
$$

with

$$
\mu a_{1}, \mu a_{2}, \mu a_{3}, \mu l, \quad(\mu>0),
$$

the probability (15) remains unchanged.

\section{Second extension}

Denoting by $S_{0}$ the lateral surface area $L_{0}$ of the parallelepiped $P_{0}$ we have that

$$
S_{0}=2\left(a_{1} a_{2}+a_{2} a_{3}+a_{1} a_{3} \sin \lambda\right)
$$

and if $S_{0}^{*}$ is the lateral surface area $L_{0}^{*}$ of the parallelepiped $P_{0}^{*}$, we have that

$$
\begin{gathered}
S_{0}^{*}=\left[2\left(a_{1}-l \cos \theta\right)\left(a_{2}-l \sin \theta \cos \varphi\right)+\right. \\
\left(a_{2}-l \sin \theta \cos \varphi\right)\left(a_{3}-l \sin \theta \sin \varphi\right)+ \\
\left.\left(a_{1}-l \cos \theta\right)\left(a_{3}-l \sin \theta \sin \varphi\right) \sin \lambda\right] .
\end{gathered}
$$

Denoting by $M_{2}$ the set of all segments $s$ which are in one of the two planes which dtermine the parallelepiped $P_{0}$ and which have the center in one of these piani. Denote likewise by $N_{2}$ the set of all segments $s$ in completely contained in lateral surface of $P_{0}$. In view of [8]:

$$
P_{\text {int }}=1-\frac{\mu\left(N_{2}\right)}{\mu\left(M_{2}\right)} .
$$

By (11), (12) and (16) we have that

$$
\mu\left(M_{2}\right)=\int_{0}^{2 \pi} d \psi \int_{0}^{\pi / 2} d \varphi \int_{0}^{\pi / 2} \sin \theta d \theta \iiint_{\left\{(x, y, z) \in L_{0}\right\}} d x d y d z=
$$




$$
2 \pi \int_{0}^{\pi / 2} d \varphi \int_{0}^{\pi / 2}\left(S_{0} \sin \theta\right) d \theta=2 \pi^{2}\left(a_{1} a_{2}+a_{2} a_{3}+a_{1} a_{3} \sin \lambda\right) .
$$

In the same way and by (17) we have that:

$$
\begin{gathered}
\mu\left(N_{2}\right)=\int_{0}^{2 \pi} d \psi \int_{0}^{\pi / 2} d \varphi \int_{0}^{\pi / 2} \sin \theta d \theta \iiint_{\left\{(x, y, z) \in L_{0}^{*}\right\}} d x d y d z=2 \pi \int_{0}^{\pi / 2} d \varphi \int_{0}^{\pi / 2}\left(S_{0}^{*} \sin \theta\right) d \theta= \\
2 \pi^{2}\left(a_{1} a_{2}+a_{2} a_{3}+a_{1} a_{3} \sin \lambda\right)- \\
l\left[a_{1}(1+\sin \lambda)+a_{2}\left(1+\frac{\pi}{2}\right)+\frac{\pi}{2} a_{3}(1+\sin \lambda)\right]-\frac{l^{2}}{2}(2-\sin \lambda) \cdot(20)
\end{gathered}
$$

By the (18), (19) and (20) we obtain that:

$$
\begin{gathered}
P_{\text {int }}=\frac{l}{2 \pi^{2}\left(a_{1} a_{2}+a_{2} a_{3}+a_{1} a_{3} \sin \lambda\right)} . \\
{\left[a_{1}(1+\sin \lambda)+a_{2}\left(1+\frac{\pi}{2}\right)+\frac{\pi}{2} a_{3}(1+\sin \lambda)+\frac{l}{2}(2-\sin \lambda)\right] .}
\end{gathered}
$$

Replacing $a_{1}, a_{2}, a_{3}$, with $\mu a_{1}, \mu a_{2}, \mu a_{3}, \mu l,(\mu>0)$, the probability (21) remains unchanged. became

Finally, for $\lambda=\pi / 2$, i.e. for a right parallelepiped, the probability (21)

$$
P_{\text {int }}=\frac{l}{2 \pi^{2}\left(a_{1} a_{2}+a_{2} a_{3}+a_{1} a_{3}\right)}\left[2 a_{1}+a_{2}\left(1+\frac{\pi}{2}\right)+\pi a_{3}+\frac{l}{2}\right] .
$$

\section{References}

[1] Badger L, Lazzarini's lucky approximation of $\pi$, 1994 MAth. Mag. 67-83.

[2] Blaschke, W.: Vorlesungen über Integralegeometrie, 3 ed., V.E.B. Deutscher Verlag der Wiss., Berlin, 1955.

[3] Buffon G., Editor's note concerning a lecture given 1733 by Mr. le Clerc de Buffon to the Royal Academy of Sciences in Paris, 1733 Hist. Acad. R. Sci. $43-45$.

[4] Buffon G., Essai d'arithmétique morale, 1777 His. Nat. Gen. Part. 4, 46123. 
[5] Laplace P-S, 1812 Théorie Analytique des Probabilité (Paris: Veuve Courcier)

[6] Laplace P-S, 1820 Théorie analytique des probabilités 3rd edn (Paris: Veuve Courcier)

[7] Pournin L, Weber M, Tsukahara M, Ferrez J. A., Ramaioli M. and Libling Th M, Three-dimensional distinct element simulation of spherocylinder crystallization, 2005 Granul. Matter 7119.

[8] Stoka M.: Une extension du problème de Buffon dans l'espace Euclidien $R^{n}$, Bollettino U.M.I. (4), 10 (1974). pp 386-389.

Received: June 3, 2014 